\title{
All-additive Inkjet Printed Humidity and Temperature Sensors Fabricated and Encapsulated at Foil Level
}

\author{
F. Molina-Lopez, A. Vásquez Quintero, G. Mattana, D. Briand, N. F. de Rooij \\ Ecole Polytechnique Fédérale de Lausanne (EPFL), Institute of Microengineering (IMT), Sensors, \\ Actuators and Microsystems Laboratory (SAMLAB), Rue Jaquet Droz 1, P.O. Box 526, CH-2002 \\ Neuchâtel, Switzerland \\ francisco.molinalopez@epfl.ch
}

\begin{abstract}
We present the simultaneous fabrication at foil level of ambient relative humidity (R.H.) and temperature sensors printed on flexible substrate. These sensors are based on capacitors and resistors and their combination allows the compensation of the R.H. signals variations at different temperatures. The whole fabrication of the system is carried out at foil level and involves the utilization of additive methods, namely inkjet printing and electrodeposition, as well as the final encapsulation of the sensors for protection. The sensors have been characterized and their performances analyzed. The sensitivity of the humidity sensor ranged from $1 \mathrm{fF} / 1 \%$ R.H. in differential mode operation to 3.5 fF / 1\% R.H. in single mode actuation. The thermal coefficient of temperature (TCR) of the thermoresistor was $4.3 \times 10^{-3}{ }^{\circ} \mathrm{C}^{-1}$. This work demonstrates the potential of inkjet printing in the fabrication at foil level of flexible, light-weight and cost-efficient large arrays of gas sensors.
\end{abstract}

Key words: Inkjet, additive, polymeric foil, flexible encapsulation, humidity capacitive sensor, thermoresistor.

\section{Introduction}

The results of this work envisage the inclusion of new functionalities on flexible RFID tags for the development of new applications, such as smart packaging, by the utilization of printed methods on polymeric foil. Compared with standard microfabrication techniques, printed and polymeric systems involve improved material and cost efficiency, better compatibility with organic materials and less demanding fabrication processes. In this work, the authors demonstrate the simultaneous development of printed humidity and temperature sensors on polyethylene terephthalate (PET) foil. The sensors are designed using in-plane interdigitated electrodes capacitors (for R.H. detection) and meander-shaped resistors (for temperature detection) as shown in Fig. 1. Such humidity sensors include a polymeric layer which absorbs water molecules, converting changes in humidity levels into capacitance variations. The resistors rely on the linear variation of electrical resistance with changes in temperature shown by some metals. Temperature control is essential in humidity sensing to account for the dependency of sorption processes with temperature. Compared with previous works dealing with the same kind of temperature [1,2] and humidity [3] sensors on flexible substrate, the sensors here have been printed on flexible polymeric substrates and encapsulated by lamination techniques at foil level. Every step in the sensors fabrication process is thus additive. Among the different existing printing technologies, inkjet has been selected due to its mask-less, contactless and localized character.

The design, fabrication (by inkjet printing and electrodeposition) and characterization of encapsulated inkjet-printed humidity and temperature sensors demonstrates the concept of large and flexible arrays of low-cost sensors, compatible with mass production methods.

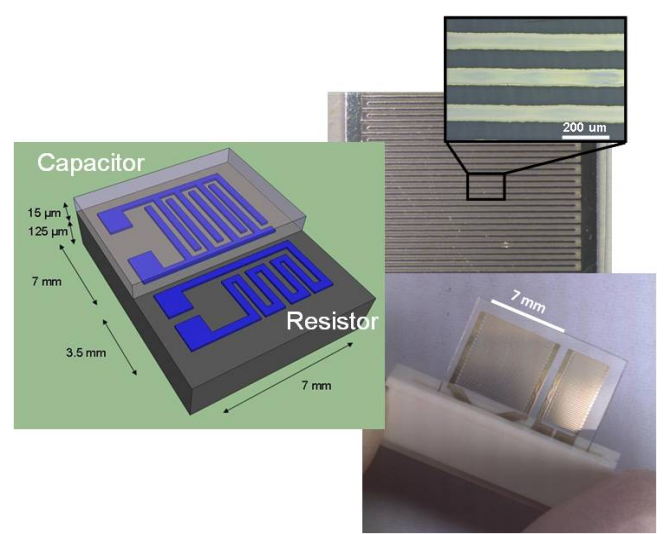

Fig. 1. Sketch and pictures of the inkjet-printed capacitors and resistors on flexible PET films. 


\section{Materials and Fabrication}

Fig. 2 depicts the main steps of the fabrication process flow with an example of a sensor structure: a) Capacitors and resistors were fabricated by ink-jet printing a commercial silver nanoparticles ink (DGP 40LT-15C from Anapro) on $125 \mu \mathrm{m}$-thick (PET) films (Melinex ST506 from Dupont) with a printer DMP2800 from Dimatix Fujifilm. The printed lines were then sintered for 30 minutes at $150^{\circ} \mathrm{C}$ in a convection oven. The fabrication of the transducers ended with electrodeposition of 1 $\mu \mathrm{m}$-thick nickel layer onto the printed lines. b) Flexible encapsulation commenced with the fabrication of rims around the sensing area using dry film photoresist by lamination at relatively low temperature $\left(85^{\circ} \mathrm{C}\right)$. c) To functionalize the capacitors for R.H. sensing, a solution of cellulose acetate butyrate (CAB) in hexyl acetate was also inkjetted onto the sensing area. More details about the fabrication of the R.H. sensors can be found in [4]. d), e) The encapsulation of the array was completed by fixation of a $135 \mu \mathrm{m}$-thick highly hydrophobic gas permeable membrane (Versapor ${ }^{\circledR}$ 10000R from PALL). Fixation between dry photoresist and Versapor membranes was performed at room temperature using a pre-patterned $50 \mu \mathrm{m}$ thick dry adhesive (ARClear 8932 from Adhesives Research).

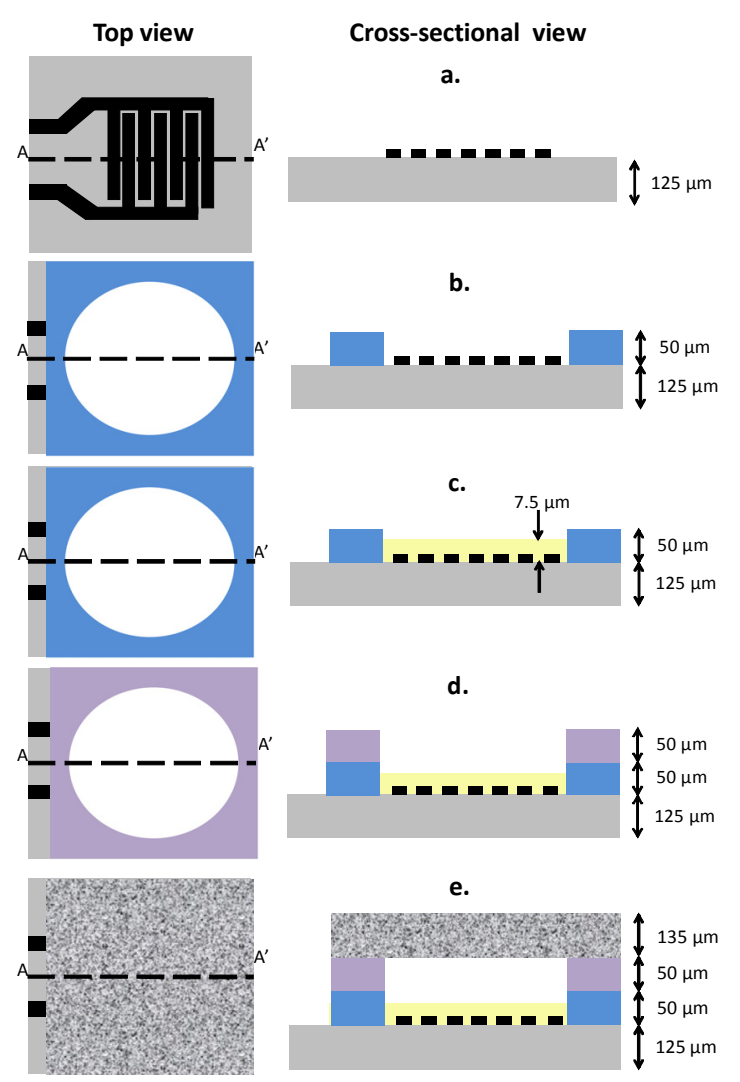

Fig. 2. Top and cross-sectional view of the process flow at foil level and sensor structure.
Fig. 3 shows the top and bottom pictures of an array of encapsulated R.H. sensors on PET.

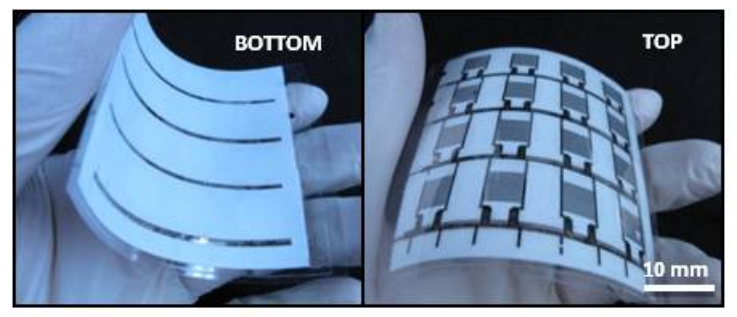

Fig. 3. Bottom- and top-view pictures of an array of inkjet-printed humidity sensors encapsulated at foil level.

\section{Sensors Design}

Interdigitated planar electrodes capacitors and meander shaped resistors with the dimensions indicated in Fig. 1 (left) composed the sensing platform. Silver printed lines had a width of 85 $\mu \mathrm{m}$ and a pitch of $115 \mu \mathrm{m}$. After silver sintering, a layer thickness of $100 \mathrm{~nm}$ was achieved. Electrodeposition of nickel increased the lines thickness up to $1 \mu \mathrm{m}$. Inkjet printing of 30 layers of $C A B$ solution built up $8 \mu$ m-thick sensing layers of dry $\mathrm{CAB}$ approximately. For more information about the design parameters of the unprotected sensors, refer to $[4,5]$.

\section{Characterization}

The capacitance of the humidity sensors was measured under different conditions of temperature and R.H., corresponding with typical ambient values. The temperatures ranged from $15^{\circ} \mathrm{C}$ to $45^{\circ} \mathrm{C}$ and the R.H. from $30 \%$ to $80 \%$. The limitations in the minimum temperature and R.H. were imposed by the operation of the climatic chamber Espec that was used to emulate different ambient conditions. Data points were taken after sensor stabilization every 30 minutes. A customized gas mixing system, allowing for the automatic control of wet and dry airflow inside a small gas cell at room temperature, served to measure the transient response of the sensors. The total air flow was $500 \mathrm{ml} / \mathrm{min}$. To eliminate the parasitic effect introduced by the polymeric substrate in the final sensor response, its signal was compared to the one of a reference device, without $\mathrm{CAB}$ coating, measured simultaneously next to the coated device. The employed frequency was $100 \mathrm{kHz}$, compatible with typical read out electronic circuits. The characterization of the thermoresistors was done in the same climatic chamber by registering the changes in resistance with temperature through the four wires method.

Multiple stacks of polymeric layers increase the possibility of interface delamination, which is 
one of the most important issues concerning the mechanical reliability of flexible sensors (when subject to bending forces). Delamination might originate from adhesion failure, cohesive failure or a combination of both. In this work, the adhesion characterization was performed using the standard ASTM D1876, known as Tpeel test. The adhesion performance of each interface within the laminated stack was evaluated and compared to optimize its process flow. Individual strips of materials, with a width of $10 \mathrm{~mm}$ and a length of $60 \mathrm{~mm}$, were tested. The force required to peel off the strips was measured with a pull-tester (Instron 3300). The lowest adhesion value was found to be between the dry film photoresist and the Versapor membrane. The latter interface was optimized by using surface treatment (oxygen plasma) on the membrane previous to lamination, which improved adhesion by a factor of two (0.12 \pm $0.04 \mathrm{~N} / \mathrm{mm}$ ).

\section{Results and Discussion}

After printing, the resistors designed as exposed before presented a resistivity of 100 $\mu \Omega^{*} \mathrm{~cm}$. This resistivity went down to $14 \mu \Omega^{*} \mathrm{~cm}$ after electrodeposition. Capacitors coated with $\mathrm{CAB}$ and protected measured 5.12 $\pm 0.19 \mathrm{pF}$. Electroplated lines capacitors did not show a significant increase in capacitance, but their signal resulted much more stable against relative humidity levels [4].

Fig. 4 presents the evolution with time of a coated capacitor signal for different R.H. steps at different temperature conditions. The increase of capacitance with R.H. and temperature occurs through the change in dielectric permittivity of the sensing layer and substrate when they absorb water. According to the work in [6], the effective permittivity of the composite sensing polymer/polar analyte (such

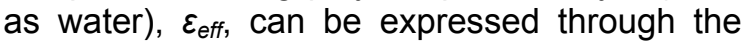
following simplified equation:

$\frac{\varepsilon_{e f f}-1}{\varepsilon_{\text {eff }}+2}=\left(1-Q \varphi_{a}\right) \frac{\varepsilon_{\text {poly }}-1}{\varepsilon_{\text {poly }}+2}+\varphi_{a} \frac{\varepsilon_{a}-1}{\varepsilon_{a}+2}$

with $\varepsilon_{\text {poly }}$ and $\varepsilon_{a}$ being the permittivity of the polymer and the analyte respectively, $\varphi_{a}$ the volume fraction of the analyte in the polymer and $Q$ a non-ideality swelling factor. $\varphi_{a}$ can be expressed as a function of the partial pressure of the analyte, $P_{a}$, the universal gas constant $R$, the absolute temperature $T$, the molar mass and the density of the analyte in liquid state, $(M$ and $\rho$ respectively), as:

$\varphi_{a}(T)=\frac{p_{a}}{R T} K \frac{M}{\rho}$
The partition coefficient $K$ needs to be calculated experimentally. According to eq. (3), $\varphi_{a}$ is temperature dependent (note that $K$ and $P_{a}$ also change with temperature). $K$ depends on the analyte concentration too, especially for high concentration values, which is the case of ambient humidity.

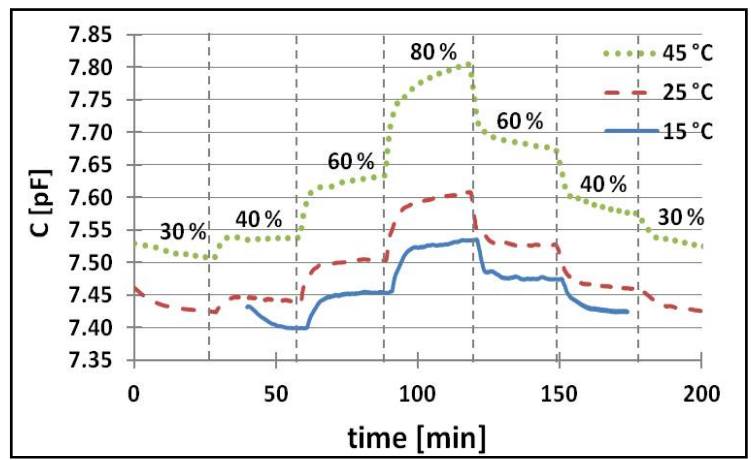

Fig. 4. Evolution with time of a coated capacitor signal for different R.H. steps at different temperatures.

Signals shown in Fig. 4 are not saturated, meaning that the chemical equilibrium in the system is not reached and the molecules of water are progressively diffusing into the substrate. Therefore, the temperature dependent diffusion coefficient of water in the sensing material, $D(T)$, plays a role in the capacitive signal too. This parasitic influence of the substrate can be partially canceled through differential measurements: Fig. 5 depicts the evolution of capacitance (in a differential mode) for the same kind of devices of Fig. 4 at room temperature. It can be observed that the signal is fairly stable at the end of every time step and does not present hysteresis. This behavior implies a quick response time calculated to range from 20 to $25 \mathrm{sec}$. Assuming the R.H. detection mechanism based in physisorption, the response time of the sensor can be estimated as [7]:

$\tau=\frac{h^{2}}{25 \pi^{2} D}$

where $D$ is the diffusion coefficient and $h$ is the thickness of the sensing layer. Hence, the quick response found in Fig. 5 corresponds exclusively with the thin $C A B$ sensing film. Special care has to be taken for proper differential operation, since it is limited to a certain range of temperatures and R.H. levels at which the partition coefficient of substrate and sensing layer is similar.

The sensitivity of the humidity sensors was about $1 \mathrm{fF} / 1 \%$ R.H. for differential mode and $3.5 \mathrm{fF} / 1 \%$ R.H. for single mode of operation. 


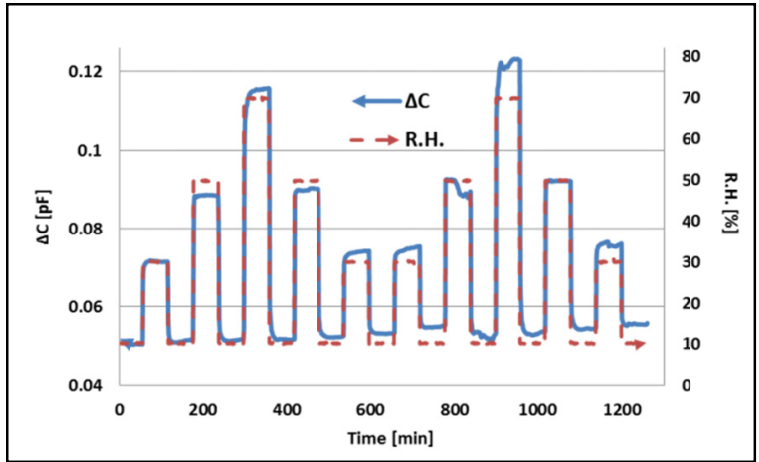

Fig. 5. Differential capacitance value versus time for a humidity sensor at room temperature. The shifts in capacitance correspond with changes in R.H. level registered with a gas flow controller.

In any case, differential or single mode, the aforementioned variations of sensor signal with temperature need to be properly understood for every concrete case of analyte / sensing material. For practical applications, variations in temperature need to be compensated through a thermometer placed next to the sensor. The fabrication of the thermometer and humidity sensor is done in parallel using the same materials and process. The thermometer also benefits from the electroplating step: Fig. 6 shows the values of normalized resistance versus temperature for bare printed silver and printed / $\mathrm{Ni}$ electroplated resistors. It can be observed how both temperature coefficient of resistance (TCR) and linearity, $R$, improved after plating from $6.3 \times 10^{-4}{ }^{\circ} \mathrm{C}^{-1}$ and 0.993 to $4.3 \times 10^{-3}{ }^{\circ} \mathrm{C}^{-1}$ and 0.999 respectively.

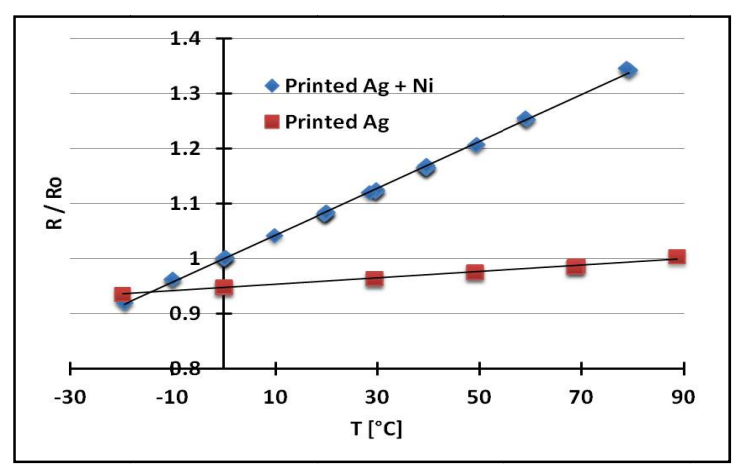

Fig. 6. Normalized resistance versus temperature for bare silver and silver-electroplated nickel resistors.

\section{Conclusions}

In this communication, we presented the integration of flexible relative humidity (R.H.) and temperature sensors on the same substrate. We demonstrated the possibility to fabricate the whole system at foil level utilizing exclusively additive printing methods. Both the relative humidity and temperature sensors were fully characterized, clearly showing that inkjet printing, combined with a foil level fabrication process, can be successfully employed for the realization of flexible, light-weight and costefficient large arrays of gas sensors.

\section{Acknowledgements}

This work is funded by the EU FP7 project FlexSmell, a Marie-Curie Initial Training Network (ITN), under the Grant no. 238454. The authors thank Dr. Francis Cardot from CSEM Neuchâtel (Switzerland) for the electrodeposition step, Dr. Bill A. MacDonald from DuPont, Dr. René Wiederkehr from PALL and Dr. Nikki Birkbeck from Adhesives Research for supplying the plastic foils used throughout the fabrication.

\section{References}

[1] Y. Moser, M. A. M. Gijs, Miniaturized Flexible Temperature Sensor. Journal of Microelectromechanical Systems 16 (6), 13491354 (2007); doi: 10.1109/JMEMS.2007.908437

[2] Oprea A., et al., Temperature, humidity and gas sensors integrated on plastic foil for low power applications. Sensors and Actuators B: Chemical $140 \quad$ (1), 227-232 (2009); doi: 10.1016/j.snb.2009.04.019

[3] A. Oprea, et al. Capacitive Humidity Sensors on Flexible RFID Labels. Sensors and Actuators B: Chemical 132 (2), 404-410 (2008); doi: 10.1016/j.snb.2007.10.010

[4] F. Molina-Lopez, D. Briand, N.F. de Rooij. All additive inkjet printed humidity sensors on plastic substrate. Sensors and Actuators B: Chemical; doi: 101016/jsnb201202042, (0)

[5] F. Molina-Lopez, J. Courbat, D. Briand, N.F. de Rooij. Inkjet Printing of Silver on Flexible Substrates for Sensing Applications. Proceedings of LOPE-C 2:011; 28-30 June, 2011; Frankfurt, Germany. pp. 278-282

[6] A.M. Kummer, A. Hierlemann, H. Baltes. Tuning Sensitivity and Selectivity of Complementary Metal Oxide Semiconductor-Based Capacitive Chemical Microsensors. Analytical Chemistry 76 (9), 2470-2477 (2004); doi: 10.1021/ac0352272

[7] R. Igreja, C.J. Dias. Dielectric response of interdigital chemocapacitors: The role of the sensitive layer thickness. Sensors and Actuators B: Chemical 115 (1), 69-78 (2006); doi: 10.1016/j.snb.2005.08.019 